\title{
Continuous caudal epidural infusion for surgical treatment of severe forms of hypospadias in pediatric patients
}

\author{
Dario Galante', Flavio Badii', Ettore Melai3, Dino Pedrotti ${ }^{4}$, \\ Massimo Savino Lambo5, Livia Cococcia ${ }^{6}$
}

\begin{abstract}
1 University Department of Anesthesia and Intensive Care, University Hospital Ospedali Riuniti of Foggia, Italy
2Department of Anesthesia and Intensive Care, Hospital of Vittorio Veneto, Italy

3Department of Anesthesia and Intensive Care, Ospedale Unico della Versilia, Lido di Camaiore, Italy

${ }^{4}$ Department of Anesthesia and Intensive Care, S. Chiara Hospital, Trento, Italy

${ }^{5}$ Department of Anesthesia and Intensive Care, Spirito Santo Hospital, Pescara, Italy

6Department of Anesthesia and Intensive Care, SS Annunziata Hospital, Sulmona, Italy
\end{abstract}

\section{Background and Goal of Study}

Severe forms of hypospadias are an important chapter of pediatric surgery. Blended anesthesia represents the best the best technique for these patients which provides excellent intra and postoperative analgesia. We evaluated the use of continuous caudal epidural infusion of levobupivacaine and fentanyl combined with general anesthesia for severe hypospadias surgery in children.

Materials and Methods

A systematic multicentric review of our recorded data was analysed. Forty-two patients, 2-6 years age, weighing $3-18 \mathrm{~kg}$, ASA II, suffering from severe forms of hypospadias were enrolled. Anaesthetic management included a combined caudal epidural and general anesthetic technique. Epidurally initial bolus $1 \mathrm{ml} / \mathrm{kg}$ of $0.25 \%$ levobupivacaine with fentanyl $1 \mu \mathrm{g} / \mathrm{kg}$ was administered after the induction of general anesthesia with a mixture of $\mathrm{Air} / \mathrm{O}_{2}$, sevoflurane and orotracheal intubation without curarization. Postoperative analgesia was maintained by continuous infusion of $0.0625 \%-0.125 \%$ levobupivacaine (depending on the age of the children) at $0.1 \mathrm{ml} / \mathrm{kg} / \mathrm{hr}$ with fentanyl $1 \mu \mathrm{g} / \mathrm{kg}$ for 2 days. Postoperative pain was assessed with FLACC pain scale using face, legs, activity, cry and consolability and sedation score was assessed by using a four point sedation score.

\section{Results}

Epidural caudal anesthesia was performed in all patients without complications. In 4 patients it was difficult to locate the caudal space and has made necessary to use an ultrasound-guided technique. Epidural catheter was introduced through the caudal space without difficulty and the height of the tip of the catheter were verified with the use of ultrasound. Children showed hemodynamic stability and only in one patient occasional bradycardia occurred. Sedation score was $<2$ and FLACC pain scale was $<4$ (Figures 1,2). Intraoperative and postoperative analgesia was excellent in all patients. Only in 3 patients, during the postoperative time, a rescue dose of ketorolac and paracetamol was necessary.

\section{Conclusions}

General anesthesia combined with epidural caudal continuous analgesia improve intra and postoperative pain control in pediatric patients undergoing severe forms of hypospadias in pediatric patients without complications or side effects.

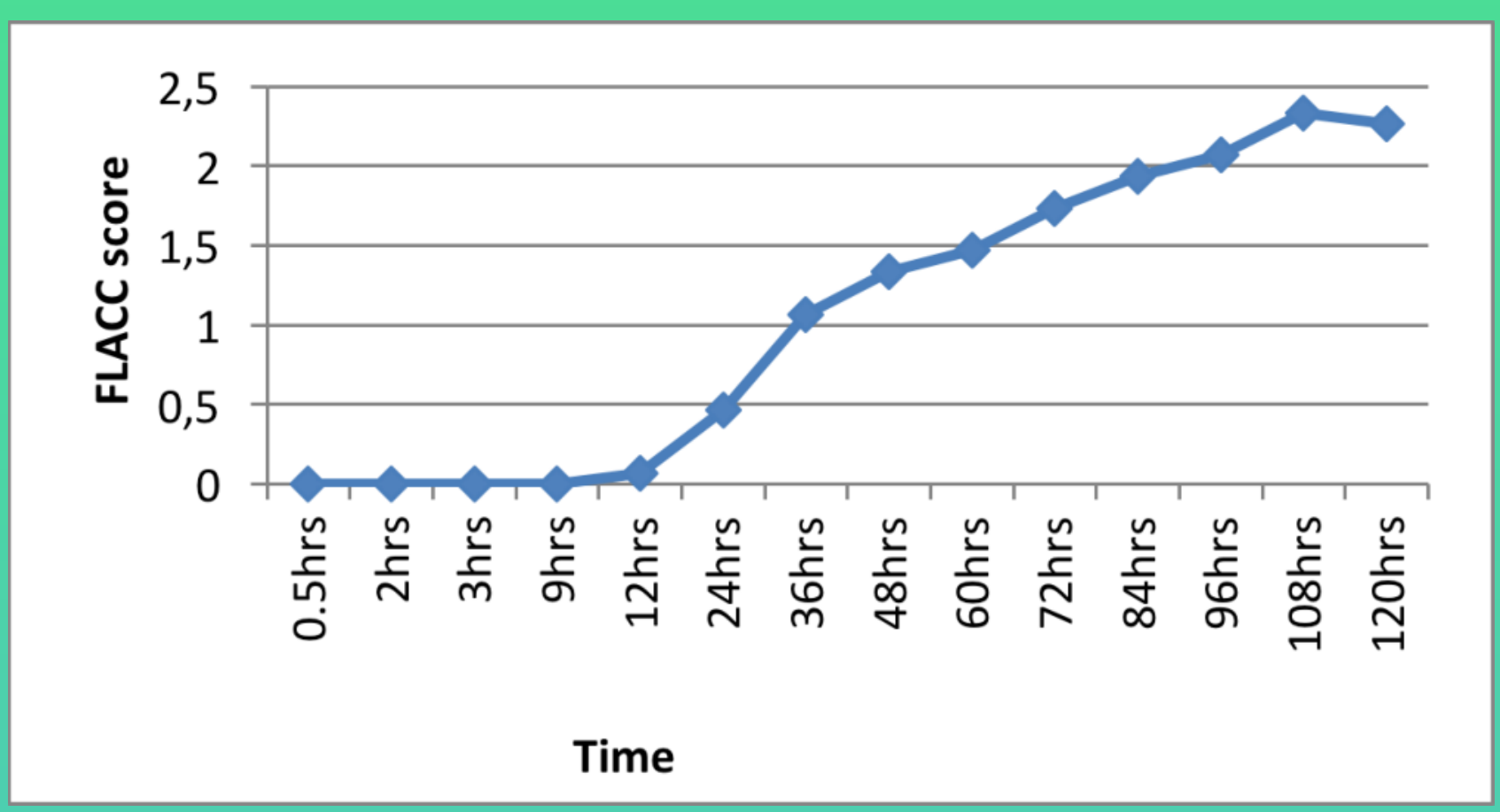

Figure 1. FLACC score in all patients was less than 4

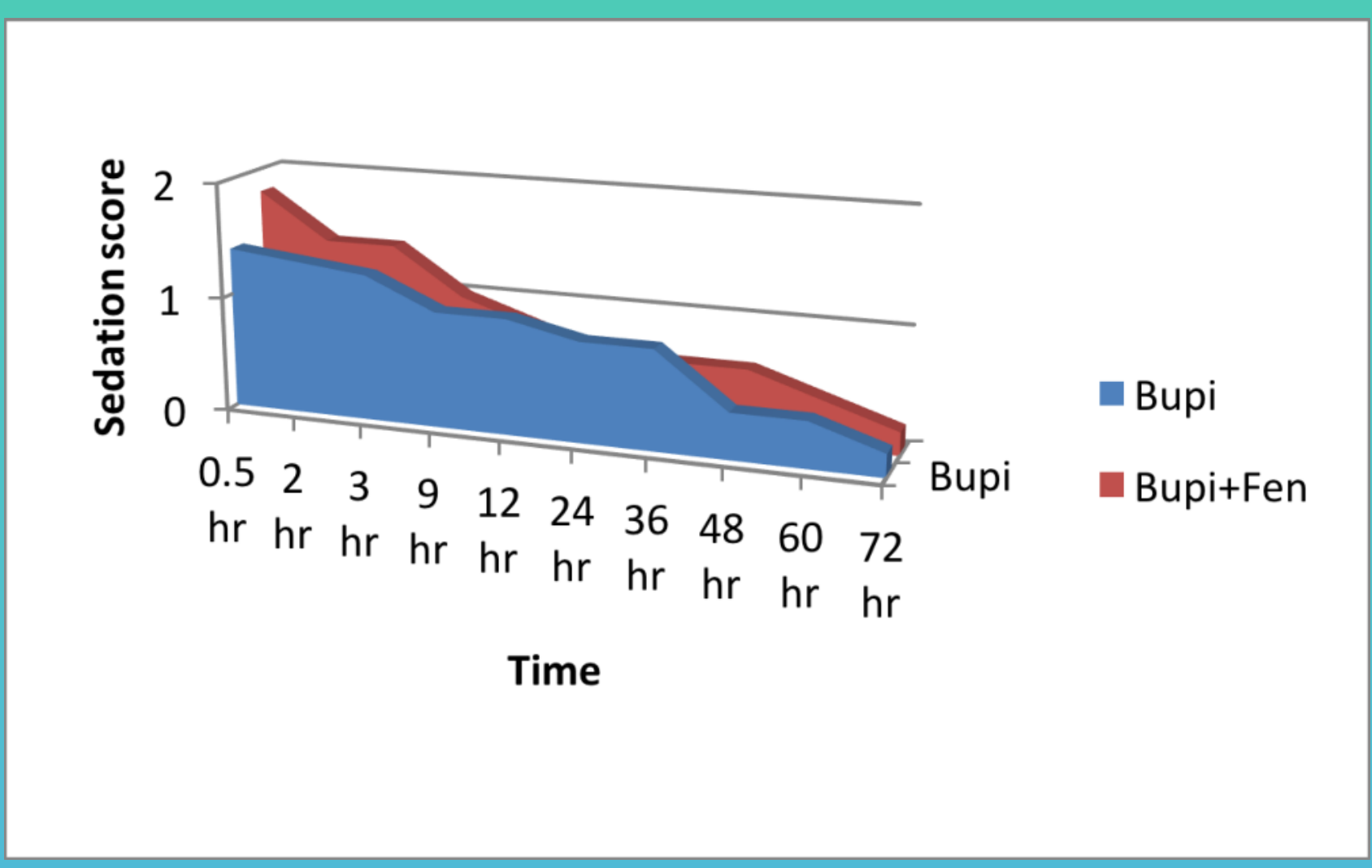

Figure 2. Sedation Score. Postoperative mean sedation score in all patients was less than 2
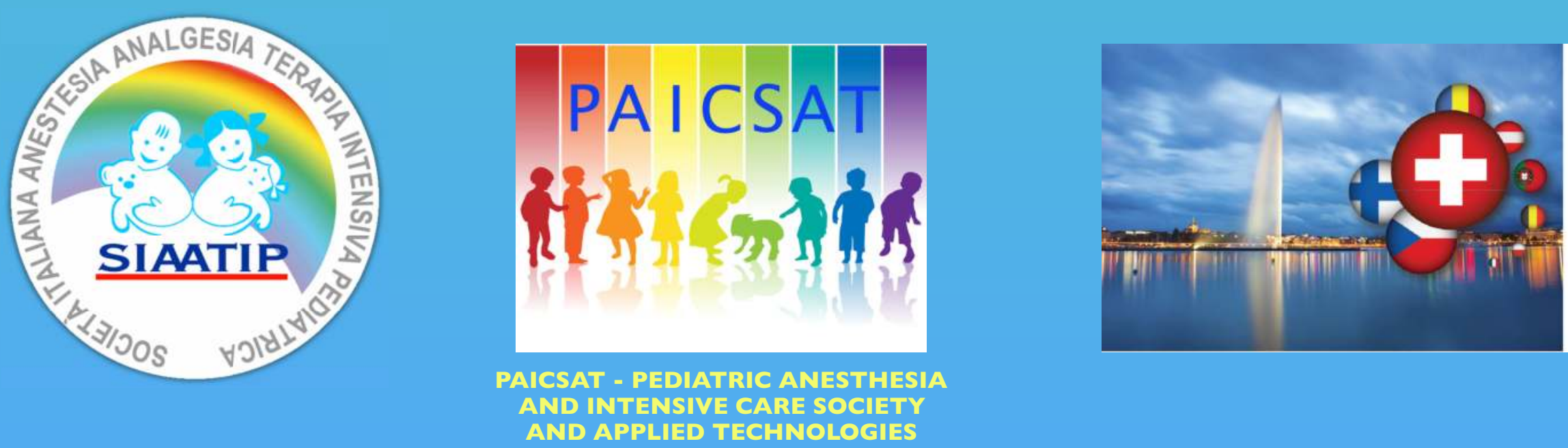